\title{
Impact of Oral Health on The Quality of Life of Elementary School Teachers
}

\author{
Folake B. Lawal ${ }^{1}$, Juliana O. Taiwo ${ }^{1}$, Gbemi A. Oke ${ }^{1}$
}

\begin{abstract}
BACKGROUND: The success of preventive school health programs is largely dependent on teachers who are role models to pupils and pivotal to implementation. It is therefore important to understand the perception of school teachers about the impact of oral health on their daily activities. The aim of this study was to assess oral health related quality of life of elementary school teachers by evaluating the impact of oral conditions like dental caries and periodontal disease on their daily performances and school work.

METHODS: This was a descriptive cross sectional study in which 407 teachers were selected through multistage random sampling technique. Data were collected with structured interviewer-administered Oral Impact on Daily Performances (OIDP) questionnaires and by clinical oral examinations. Data were collated and analysed with SPSS.

RESULTS: The prevalence of reporting of impacts on daily performances was $39.1 \%$ with eating and enjoying food being the most commonly impacted activity. Impacts on daily performances were more likely to be reported by teachers with better education $(p=0.019)$. Teachers with caries had higher odds of reporting impacts as a result of oral health status (CI: 1.04, 5.64, $p=0.040)$. The severity of periodontal disease found on clinical examination was not related to reporting of impacts on quality of life $(p>0.05)$.

CONCLUSIONS: The oral health status of school teachers impacted significantly on their daily performance and school work when they experienced pain. Painless but highly prevalent oral lesions were, however, not perceived to influence their quality of life.
\end{abstract}

KEYWORDS: Oral health status, OIDP, quality of life, school-based program, teachers

DOI: http://dx.doi.org/10.4314/ejhs.v25i3.4

\section{INTRODUCTION}

Oral Health Related Quality of Life (OHRQoL) measure evaluates dimensions of oral health such as psychological, social and emotional conditions that are not routinely assessed by clinical measures $(1,2)$. All the instruments used in measuring OHRQoL have been found useful in terms of the number of dimensions and have been validated worldwide (1-6). Although these instruments have been used on different subpopulations to determine oral health related quality of life, there is very little information about their usage on specific workforce such as school teachers who are involved in promotion of school health.
Teachers play important roles as models for the school pupils and are the fulcrum for the implementation of school based oral health programs $(7,8)$. The school based oral health promotion program creates an avenue in which positive long term behavior is maintained in the pupils $(9,10)$. The success of this program has been found to be linked to the input of teachers in the schools, with positive results when the teachers' attitude is supportive of oral health promotion $(7,8)$.

Regrettably, it is nonexistentin many developing countries, where high prevalence of preventable oral diseases exists; with dental caries as the leading cause of tooth loss present in $25 \%$

\footnotetext{
${ }^{1}$ Department of Periodontology and Community Dentistry, University of Ibadan, Ibadan, Nigeria Corresponding Author: Dr. F.B. Lawal, Email:folakemilawal@yahoo.com
} 
of children, and over $70 \%$ require periodontal treatment (11).

The failure to organize school based oral health programs may include poor knowledge of oral health that exists among teachers in developing countries (12), or that they are not bothered about their oral health status since it does not affect their daily activities. The latter has not been investigated, which if done, may lead to a better understanding of the impact of oral health on the daily activities of school teachers. This thus necessitates the need to assess their OHRQoL, which characterises an individual's perception of oral health and how it impacts on daily activities. The fact that these perceptions can act as variables that influence activities related to the teachers' health and that of their pupils, and will also help in understanding the value teachers assign to oral health, further necessitated this study.

The aim of the study, therefore, was to assess the oral health related quality of life of elementary school teachers in Ibadan, Nigeria, using the Oral Impacts on Daily Performances (OIDP) inventory. The Oral Impacts on Daily Performances (OIDP) focuses on measuring the implication of oral symptoms on the individual's ability to perform daily activities. It is short and easy to use; and it has the advantage of measuring behavioral impacts on performances (13), which makes it an appropriate tool for this study. The major hypothesis that guided the study was that the presence of oral diseases e.g. dental caries and periodontal disease in school teachers do not affect their daily performances and school work.

\section{MATERIALS AND METHODS}

Study design and location: This was a descriptive cross sectional study conducted amongst elementary school teachers (Grades I to VI) in Ibadan, Nigeria, between April and July, 2011. Ibadan is the largest indigenous city in SubSaharan Africa covering a land area of 3,123 square kilometers and has an estimated population of over 5 million inhabitants.

Sampling technique and selection: A minimum of 384 teachers was calculated to be necessary for the study using a prevalence of impact on daily activities, from a previous study, of $51 \%$ (6), a precision (d) of 5\% and confidence interval of 95\% with the Kish-Leslie formula for crosssectional studies (14). Teachers in the study were selected using a multi-stage random sampling technique. The first stage involved the selection of three local government areas (from the eleven local government areas in Ibadan) through simple random sampling technique. In the second stage, one school zone from each of the three selected local government areas was selected by simple random sampling. All the public elementary schools (31) within the three selected zones were included in the study. All the teachers in the selected schools who gave consent were included in the study.

Privately operated elementary schools were not included in the study because the State Universal Education Board did not have a full list of the approved private elementary schools under its jurisdiction at the time of the study. The exclusion was done to avoid selection bias.

Data collection procedure: Ethical approval was obtained from the Institution's Ethical Review Committee. Data was collected with a structured interviewer administered questionnaire and by clinical oral examination. The questionnaire assessed the sociodemographic characteristics of the teachers and the impacts of oral health on daily performances using the OIDP inventory.

Oral impact on daily performances measure: This was assessed using the frequency and the severity scales of the OIDP. The OIDP frequency items were assessed by asking 'During the past 6 months, how often have problems with your mouth and teeth caused you any difficulty with: eating and enjoying food, speaking and pronouncing clearly as to affect communication with the school pupils or teaching them, cleaning teeth, sleeping and relaxing, smiling and showing teeth without embarrassment as to affect school work, maintaining usual emotional state, carrying out daily school task (which included teaching of the school pupils, counseling the school pupils and other school activities) and social role, and enjoying contact with the pupils and other teachers?'

Each question was assessed using a 6-point scale in the range: $0=$ 'never', $1=$ 'affected less often than once a month (e.g. once in six months)', $2=$ 'once or twice a month', $3=$ 'once or twice a week', $4=$ ' 3 to 4 times a week' and $5=$ 'every day' (i.e. lower scores reflected less frequent impacts on daily activities). The affected daily performance was also classified according to the 
degree of severity as $0=$ 'none', 1 = 'very little', 2 $=$ 'little', $3=$ 'moderate', $4=$ 'severe', and $5=$ 'very severe'. The total OIDP score was calculated by multiplying the frequency and severity score of each item. A pre-test was conducted on twenty teachers from a school not included in the study and the OIDP inventory evaluated for its psychometric properties.

Oral examination: A researcher (FBL) who had been trained and calibrated, assisted by a trained recorder, examined all the teachers. Oral examination was carried out using gloves, sterile dental mirror, wooden spatula and periodontal probe. Measurement with these instruments was in accordance with the WHO criteria (15).

The dental caries experience was assessed using the Decayed, Missing and Filled (DMFT) index. The DMFT score was derived from the sum of the individual components of the DMFT. Periodontal disease was evaluated using the CPITN index (16). Details of the periodontal health of the teachers is the subject of another study (17).

Intra-examination variability: Intra-examiner variability was calculated for correct and standard measurements: every tenth teacher had a duplicate examination until a total of $40(10 \%)$ were reexamined. The values of intra-examiner Kappa scores were 0.92 for examination for coronal caries and 0.94 for CPITN.

Data management and analysis: Data were collated and analyzed using the SPSS version 17 software. For the purpose of cross tabulation, age and teachers' years of experience were dichotomized according to the mean, sex was considered as male and female; marital status dichotomized as married and not married which included singles, separated and widowed, and salary scale was collapsed into binary variable according to the median salary grade level. For the purpose of cross tabulation and analysis, total OIDP score was dichotomized yielding the categories 'OIDP Score $=0$ ' and 'OIDP Score $\geq$ 1'; the DMFT score was reconstructed into a binary variable as either 'DMFT $=0$ ' and 'DMFT $>0$ ', and the CPITN score was dichotomized based on the presence of pathological pocketing.

Test of association was conducted between the OIDP score and sociodemographic variables using Chi-square statistics. Logistic regression was performed to identify significant predictors of
OIDP scores by inputting clinical oral examination variables into the equation, stepwise, at a threshold $p$-value of 0.10 during the crosstabulation. The $\mathrm{p}$-value for statistical significance was $<0.05$.

\section{RESULTS}

Sociodemographic characteristics: A total of 407 school teachers participated in the study. The mean age of the participants was $48.3( \pm 6.2)$

years. The mean years of experience in the teaching service was $22.9( \pm 7.8)$ years and salary grade level ranged from 6 to 14 . Two hundred and thirty-seven $(58.2 \%)$ were on grade level 13 (assistant head-teacher equivalent) or higher.

OIDP Score and frequency of impact on daily performance: A total of 159 teachers reported some impact of oral health on their daily performances (i.e. OIDP score > 0). The OIDP score of the study participants ranged from 0 to 100 with a mean of $9.64( \pm 15.5)$. The OIDP items most commonly affected by oral health status were eating and enjoying food $(28.3 \%)$, cleaning teeth $(14.5 \%)$, sleeping and relaxing $(13.0 \%)$ and speaking and pronouncing words clearly (11.3\%). The least commonly affected were carrying out daily school task (7.9\%), maintaining usual emotional status (7.6\%), smiling and showing teeth $(7.1 \%)$ and enjoying social contact with people $(6.6 \%)$.

Oral health status of the participants: The mean DMFT score was $0.61( \pm 1.60)$ with a range of 0 to 14 . The DMFT was greater than zero (0) in 97 participants. Of the total DMFT, the largest contribution (70.3\%) was from the "M" component while the least $(5.2 \%)$ was from the "F" component. Of the study participants, $29.5 \%$ had pathological periodontal pocketing.

Oral impact on daily performance and sociodemographic characteristics: Table 1 shows the relationship between OIDP score and sociodemographic characteristics of the participants. The proportion of teachers who had university degrees and reported impacts (OIDP score $\geq 1$ ) was significantly higher than those with NCE who reported impacts $(\mathrm{p}=0.019)$. There were no statistically significant differences between OIDP score and age, gender, marital status, years of teaching or salary grade level (Table 1). 
Table 1: Relationship between OIDP score and sociodemographic characteristics

\begin{tabular}{|c|c|c|c|c|c|}
\hline \multirow[b]{2}{*}{ Variable } & \multicolumn{3}{|c|}{ OIDP score } & \multirow[b]{2}{*}{$\chi^{2}$} & \multirow[b]{2}{*}{ p value } \\
\hline & $\begin{array}{l}\geq 1 \text { - Impact present } \\
\text { No }(\%)\end{array}$ & $\begin{array}{l}\text { o- No impact } \\
\text { No }(\%)\end{array}$ & $\begin{array}{l}\text { Total } \\
\text { No }(\%)\end{array}$ & & \\
\hline \multicolumn{6}{|l|}{ Age group (years) $<48$} \\
\hline & $53(38.4)$ & $85(61.6)$ & $138(100.0)$ & 0.038 & 0.845 \\
\hline \multicolumn{6}{|l|}{ Total } \\
\hline & $106(39.4)$ & $163(60.6)$ & $269(100.0)$ & & \\
\hline & $159(39.1)$ & $248(60.9)$ & $407(100.0)$ & & \\
\hline \multicolumn{6}{|l|}{ Gender } \\
\hline Male & $11(35.5)$ & $20(64.5)$ & $31(100.0)$ & 0.181 & 0.671 \\
\hline \multicolumn{6}{|l|}{ Female } \\
\hline \multirow[t]{2}{*}{ Total } & $148(39.4)$ & $228(60.6)$ & $376(100.0)$ & & \\
\hline & $159(39.1)$ & $248(60.9)$ & $407(100.0)$ & & \\
\hline \multicolumn{6}{|l|}{ Marital status } \\
\hline Unmarried & $8(44.4)$ & $10(55.6)$ & $18(100.0)$ & 0.229 & 0.632 \\
\hline \multicolumn{6}{|l|}{ Married } \\
\hline \multirow[t]{2}{*}{ Total } & $151(38.8)$ & $238(61.2)$ & $389(100.0)$ & & \\
\hline & $159(39.1)$ & $248(60.9)$ & $407(100.0)$ & & \\
\hline \multicolumn{6}{|l|}{ Educational level } \\
\hline University & $46(49.5)$ & $47(50.5)$ & $93(100.0)$ & 5.473 & $0.019 *$ \\
\hline \multicolumn{6}{|l|}{ NCE } \\
\hline \multirow{2}{*}{ Total } & $113(36.0)$ & 201(64.0) & $314(100.0)$ & & \\
\hline & $159(39.1)$ & $248(60.9)$ & $407(100.0)$ & & \\
\hline \multicolumn{6}{|l|}{ Years of teaching } \\
\hline$\leq 23$ years & $71(37.4)$ & $119(62.6)$ & $190(100.0)$ & 0.432 & 0.511 \\
\hline \multicolumn{6}{|l|}{$>23$ years } \\
\hline \multirow[t]{2}{*}{ Total } & $88(40.6)$ & $129(59.4)$ & $217(100.0)$ & & \\
\hline & $159(39.1)$ & $248(60.9)$ & $407(100.0)$ & & \\
\hline \multicolumn{6}{|l|}{ Salary grade } \\
\hline$<13$ & $63(37.1)$ & $107(62.9)$ & $170(100.0)$ & 0.494 & 0.482 \\
\hline \multicolumn{6}{|l|}{$\geq 13$} \\
\hline \multirow[t]{2}{*}{ Total } & $96(40.5)$ & $141(59.5)$ & $237(100.0)$ & & \\
\hline & $159(39.1)$ & $248(60.9)$ & $407(100.0)$ & & \\
\hline
\end{tabular}

*Statistically significant

OIDP score of participants and oral examination findings: A higher proportion of teachers with carious teeth had OIDP score greater than zero, i.e. cariies had some impact on quality of life, compared to those without carious teeth $(61.1 \%$ vs. $36.9 \%, \mathrm{p}=0.005)$. There was no association between having missing teeth, filled teeth, DMFT score or periodontal disease and OIDP score (Table 2). 
Table 2: Relationship between OIDP score of participants and oral examination findings

\begin{tabular}{|c|c|c|c|c|c|}
\hline \multicolumn{6}{|c|}{ OIDP score } \\
\hline $\begin{array}{l}\text { Oral examination } \\
\text { findings }\end{array}$ & $\begin{array}{l}\geq 1-\text { Impact present No } \\
(\%)\end{array}$ & $\begin{array}{l}0-\text { No } \\
\text { Impact } \\
\text { No }(\%) \\
\end{array}$ & $\begin{array}{l}\text { Total } \\
\text { No }(\%)\end{array}$ & $\chi^{2}$ & $\begin{array}{l}\mathbf{p} \\
\text { value }\end{array}$ \\
\hline \multicolumn{6}{|l|}{ Decayed (DMFT) } \\
\hline Yes & $22(61.1)$ & $14(38.9)$ & $36(100.0)$ & 8.063 & $0.005^{*}$ \\
\hline No & $137(36.9)$ & $234(63.1)$ & $371(100.0)$ & & \\
\hline Total & $159(39.1)$ & $248(60.9)$ & $407(100.0)$ & & \\
\hline \multicolumn{6}{|l|}{ Missing (DMFT) } \\
\hline Yes & $34(42.0)$ & $47(58.0)$ & $81(100.0)$ & 0.359 & 0.549 \\
\hline No & $125(38.3)$ & $201(61.7)$ & $326(100.0)$ & & \\
\hline Total & $159(39.1)$ & $248(60.9)$ & $407(100.0)$ & & \\
\hline \multicolumn{6}{|l|}{ Filled (DMFT) } \\
\hline Yes & $4(44.4)$ & $5(55.6)$ & $9(100.0)$ & 0.112 & 0.738 \\
\hline No & $155(38.9)$ & $243(61.1)$ & $398(100.0)$ & & \\
\hline Total & $159(39.1)$ & $248(60.9)$ & $407(100.0)$ & & \\
\hline \multicolumn{6}{|l|}{ DMFT Score } \\
\hline$>0$ & $46(47.4)$ & $51(52.6)$ & $97(100.0)$ & 3.736 & 0.053 \\
\hline$=0$ & $113(36.5)$ & $197(63.5)$ & $310(100.0)$ & & \\
\hline Total & $159(39.1)$ & $248(60.9)$ & $407(100.0)$ & & \\
\hline \multicolumn{6}{|l|}{ Periodontal disease } \\
\hline Absence of pocketing & $114(39.7)$ & $173(60.3)$ & $287(100.0)$ & 1.705 & 0.636 \\
\hline Presence of pocketing & $45(37.5)$ & $75(62.5)$ & $120(100.0)$ & & \\
\hline Total & $159(39.1)$ & $248(60.9)$ & $407(100.0)$ & & \\
\hline
\end{tabular}

*Statistically significant

Predictors of OIDP score among oral examination findings

Table 3 shows that teachers with caries have a without caries (OR: 2.4, 95\% CI: 1.04, 5.64, p = 0.040). The DMFT status was not a significant predictor of OIDP score $(\mathrm{p}=0.669)$. greater odd of having OIDP score $\geq 1$ than those

Table 3: Logistic regression analysis of relationship between OIDP score and oral examination findings

\begin{tabular}{llllll}
\hline Variable & Categories of variable & B & OR & 95\% CI & p value \\
\hline Decayed teeth & Yes & 0.885 & 2.423 & $1.041-5.636$ & $0.040^{*}$ \\
& No** & & & & \\
DMFT Score & $>0$ & 0.123 & 1.131 & $0.644-1.986$ & 0.669 \\
& $=0^{* *}$ & & & & \\
\hline
\end{tabular}

*Statistically significant, $* *$ - Reference category for comparison

\section{DISCUSSION}

The findings of this study showed that the prevalence of impact of oral health on daily performances and school activities of the teachers was $39.1 \%$. This prevalence is lower than that reported from a study conducted among adults in
Benin City, Nigeria, where $62 \%$ said that their oral health status had impacted on their quality of life (2). The difference may be accounted for by the subjects included in that study, who were from a hospital setting, whereas those in the present study were recruited from outside the hospital setting. Masalu and Åstrøm (6) in Tanzania, Åstrøm and Okullo (3) in Uganda and Gomes et al. (4) in 
Brazil, using the same OIDP instrument, also documented higher prevalence of impact. The higher prevalence may be attributable to the age dissimilarity of the participants in the studies from Uganda and Brazil who were adolescents and adults aged 35 to 44 years in contrast to a higher mean age in the present study. On the other hand, the prevalence of impact in this study is higher than those reported from Europe $-18.3 \%$, United States $-15.3 \%$ and Australia $-15.7 \%(1,18)$. The present study thus adds to the literature that suggests that prevalence of oral impact on daily performance is higher in Africans than in Caucasians and this may be due to differences in cultural and behavioral practices (6), or still on the availability of facilities.

Difficulty with eating and enjoying food was mostly reported by the teachers as impact on daily performances resulting from their oral health status. This is in agreement with the finding of Åstrøm and Okullo (3), in 1146 Ugandan adolescents of in which $44 \%$ reported difficulty with eating and enjoying food as the leading impact on their daily performances. Similarly, $35 \%$ of adults aged 20 to 50 years (mean age of 36 years) in a study of 285 Iranians reported difficulty with eating and enjoying food as the major impact on their daily performances (19). Other authors have confirmed the predominant impact that oral health status has on eating $(1,2,6)$.

Difficulty with cleaning teeth was the second most prevalent impact identified in the present study. This being similar to the finding from the study conducted in Uganda, in which $35 \%$ of the study participants mentioned difficulty with cleaning teeth as the second most frequently impacted activity (3). Eating and cleaning teeth are more frequently impacted upon than other activities because of the importance of food and nutrition to humans; hence; high consideration is attached to the feeding process. Impairment of this activity or cleaning the teeth before or after meals is also considered very important to individuals.

Poor oral health status impacted on the ability of $8 \%$ of the teachers to perform their daily school tasks. This prevalence is slightly less than the proportion of Tanzanian students who reported that poor oral health status had interfered with their ability to carry out school work (10\%) in a study carried out by Masalu and Åstrøm (6). It is, however, higher than the proportion of Norwegian adults who reported poor oral health status as impacting on their ability to carry out major work (1). The differences may be due to the divergent nature of the major daily task of the population sampled in each of the studies.

In our study, the psychological and social impacts were less frequently reported. Maintaining usual emotional status and enjoying social contact were rarely impacted upon by the oral health status of the teachers. Similar results were published by others $(1,6)$. The low frequency of reporting psychological and social impacts may be due to the relative unwillingness to report selfconscious or embarrassing problems related to oral health challenges $(20,21)$. Perhaps, this has some bearing on the psychosocial attributes of races and cultures. Factors responsible for this behavior will need to be investigated.

Although teachers who were older, unmarried, with university education, greater teaching experience or on a higher salary scale were more likely, in this study, to report an impact on daily performances and school work. The difference was only statistically significant when highest educational attainment was compared. Teachers with university education reported impacts on daily performances and school work than their counterparts. Okunseri et al. (2) reported similar findings on the relationship between oral health related quality of life measures and sociodemographic indices of age, ethnicity and employment status as well as sex and educational level. In that study, the mean of effects score (on the OHRQoL-UK inventory) for those with secondary/tertiary education (61.8) was significantly higher than those with less than secondary/tertiary level of education (57.2). There were no statistically significant differences when age, sex, ethnicity and employment status were compared with the mean QoL effects score (2). This may reflect the influence of education on awareness of oral health and modification of health seeking behavior that is more likely expressed by those with better education in this environment.

The study also found that teachers with dental caries were more likely to report their oral condition as having impacted on their daily performances compared to those without dental caries. The pain that accompanies dental caries invariably draws the attention of the individual, 
leading therefore to significant influences on their daily performances. This corroborates with studies that have shown that most of the people, who had utilized dental services in the past did so because of toothache $(2,6,22,23)$. An alternative explanation is that adults are highly concerned with tooth functions, which are ultimately affected when dental caries, missing teeth and periodontal pockets are present (3).

Our study revealed that periodontal disease, though highly prevalent amongst the teachers, had no significant impact on their daily activities. The chronicity of the disease may account for this. It is probable that they perceived no harm with a condition when it is not associated with pain. Periodontal disease in general is notorious for being painless unless complicated. Furthermore, this oral condition has been associated with systemic diseases like diabetes mellitus and heart diseases that are known to have high incidences of morbidities (24) as well as being one of the major causes of tooth loss. Despite this, periodontal disease is highly preventable using simple oral hygiene measures, which ideally should be taught as part of the curriculum in teacher training schools. This, therefore, suggests a need to promote oral health among teachers emphasizing the importance of prevention of common oral diseases that do not significantly impact on their daily activities in order to avoid the complications associated with oral diseases. Effective oral health education and promotion workshops should be organized regularly for school teachers. The workshops should be aimed at changing the perception that oral health is disparate to general health, and emphasis should be placed on the importance of oral health and its relationship with general health.

The major limitations of this study include the recruitment of public primary school teachers, excluding those in private schools. It is not known if this could have had any bearing on the results obtained. Furthermore, the teachers were mostly in their forties and fifties, therefore it may be difficult to generalise the findings to younger adults.

In conclusion, the oral health status of school teachers impacted significantly on their daily performance and school work when they experienced pain. Painless, but highly prevalent oral lesions, were not perceived to influence the teachers' QoL. There was a significant association between the highest level of education attained by the teachers and reporting of an impact on QoL.

\section{REFERENCES}

1. Astrom AN, Haugejorden O, Skaret E, Trovik TA, Klock KS. Oral Impacts on Daily Performance in Norwegian adults: the influence of age, number of missing teeth, and socio-demographic factors. Eur J Oral Sci 2006;114:115-121.

2. Okunseri C, Chattopadhyay A, Lugo RI, McGrath C. Pilot survey of oral health-related quality of life: a cross-sectional study of adults in Benin City, Edo State, Nigeria. BMC Oral Health 2005;5:7.

3. Astrom AN, Okullo I. Validity and reliability of the Oral Impacts on Daily Performance (OIDP) frequency scale: a cross-sectional study of adolescents in Uganda. BMC Oral Health 2003;3:5.

4. Gomes AS, Abegg C, Fachel JM. Relationship between oral clinical conditions and daily performances. Braz Oral Res 2009;23:76-81.

5. Lawal FB, Taiwo JO, Arowojolu MO. Validation of the oral impact on daily performance frequency scale in Ibadan, Nigeria. Afr J Med Med Sci 2013;42:215-222.

6. Masalu JR, Astrom AN. Applicability of an abbreviated version of the oral impacts on daily performances (OIDP) scale for use among Tanzanian students. Comm Dent Oral Epidemiol 2003;31:7-14.

7. Gill P, Chestnutt IG, Channing D. Opportunities and challenges to promoting oral health in primary schools. Community Dent Health 2009;26:188-192.

8. Stokes E, Pine CM, Harris RV. The promotion of oral health within the Healthy School context in England: a qualitative research study. BMC Oral Health 2009;9:3.

9. Araojo J. Philipines Country Report: School oral health promotion programme. In, The 2 nd Asian Conference of Oral Health Promotion for School Children Prospectus for Our Future Generation; 2003.

10. Kwan SY, Petersen PE, Pine CM, Borutta A. Health-promoting schools: an opportunity for oral health promotion. Bulletin of the World Health Organization 2005;83:677-685. 
11. Agbelusi GA, Jeboda SO. Oral health status of 12-year-old Nigerian children. West Afr J Med 2006;25:195-198.

12. Sofola OO, Agbelusi GA, Jeboda SO. Oral health knowledge, attitude and practices of primary school teachers in Lagos State. Niger J Med 2002;11:73-76.

13. Adulyanon A, Sheiham A. Oral impacts on daily performances. In: Slade GD, editor. Measuring Oral Health and Quality of Life. Chapel Hill: University of North Carolina, Dental Ecology; 1997. p. 151-160.

14. Kish L: Survey Sampling. Wiley Interscience Publication, New York; 1965.

15. Oral health surveys: basic methods, $4^{\text {th }}$ edition. World Health Organization Geneva; 1997.

16. Ainamo J, Barmes D, Beagrie G, Cutress T, Martin J, Sardo-Infirri J. Development of the World Health Organization (WHO) community periodontal index of treatment needs (CPITN). Int Dent J 1982;32:281-291.

17. Lawal FB, Dosumu EB. Periodontal status and treatment needs of primary school teachers in the absence of formal school oral health programme. Niger Dent J 2014;22:32-37.

18. Sanders AE, Slade GD, Lim S, Reisine ST. Impact of oral disease on quality of life in the US and Australian populations. Community Dent Oral Epidemiol 2009;37:171-181.

19. Dorri M, Sheiham A, Tsakos G. Validation of a Persian version of the OIDP index. $B M C$ Oral Health 2007;7:2.

20. Locker D, Miller Y. Evaluation of subjective oral health status indicators. J Public Health Dent 1994;54:167-176.

21. Locker D, Slade G. Oral health and the quality of life among older adults: the oral health impact profile. $J$ Can Dent Assoc 1993;59:830-833,837-838,844.

22. Taiwo JO, Noah M. Pattern of dental clinic attendance of registered diabetic patients in Ibadan. J Med Biomed Res 2006;5:36-43.

23. Adekoya-Sofowora C, Nasir W, Oginni A et al. Dental caries in 12-year-old suburban Nigerian school children. African Health Sciences 2006; 6: 145-150.

24. Rai B. Systemic Effect of Oral Disease. The Internet Journal of Family Practice 2007;5:1. 